NOTICE: This is the author's version of a work that was accepted for publication in Applied Mathematics and Computation. Changes resulting from the publishing process, such as peer review, editing, corrections, structural formatting, and other quality control mechanisms may not be reflected in this document. Changes may have been made to this work since it was submitted for publication. A definitive version was subsequently published in Applied Mathematics and Computation [217, 2, 2010] DOI 10.1016/j.amc.2010.05.087 


\title{
Global Exponential Stability of Impulsive Discrete-time Neural Networks with Time-Varying Delays
}

\author{
Honglei Xu, Yuanqiang Chen and Kok Lay Teo
}

\begin{abstract}
This paper studies the problem of global exponential stability and exponential convergence rate for a class of impulsive discrete-time neural networks with time-varying delays. Firstly, by means of the Lyapunov stability theory, some inequality analysis techniques and a discrete-time Halanay-type inequality technique, sufficient conditions for ensuring global exponential stability of discrete-time neural networks are derived, and the estimated exponential convergence rate is provided as well. The obtained results are then applied to derive global exponential stability criteria and exponential convergence rate of impulsive discrete-time neural networks with time-varying delays. Finally, numerical examples are provided to illustrate the effectiveness and usefulness of the obtained criteria.
\end{abstract}

\section{Index Terms}

Impulsive discrete-time neural networks, global exponential stablity, exponential convergence rate, Halanay inequality.

\section{INTRODUCTION}

Neural networks have received extensive interests in recent years and have witnessed many promising potential applications in different areas such as signal processing, content addressable

Honglei Xu is with the Department of Mathematics and Statistics, Curtin University of Technology, Perth, WA 6845, Australia (email:h.xu@curtin.edu.au).

Yuanqiang Chen is with Department of Mathematics, Guizhou University, Guiyang 550025, China.

Kok Lay Teo is with the Department of Mathematics and Statistics, Curtin University of Technology, Perth, WA 6845, Australia. 
memory, pattern recognition and combinatorial optimization (see e.g. [2]-[4], [6]-[8] and the references therein). It is well known that the existence of delays in neural networks causes undesirable complex dynamical behaviors such as instability, oscillation and chaotic phenomena. Stability problems of time-delayed neural networks have attracted much attention in view of their theoretical as well as practical importance. There are now many results being reported in the literature on neural networks with time delays, see, for example, [1]-[3], [5], [6], [8]-[17], and references therein. In practice, for computation convenience, continuous-time neural networks are often discretized to generate discrete-time neural networks. Thus, the study of discretetime neural networks attracts more and more interests. In recent years, asymptotic behaviors of discrete-time neural networks have been investigated, and many results have been reported (see [14]-[18] and the references therein).

Complex dynamical systems usually undergo abrupt changes of their states at certain moments due to unexpected internal or external effects. There is no exception for discrete neural networks. These impulsive perturbations can also cause undesirable dynamical behaviors leading to poor performance. Therefore, it is necessary to take into account both impulsive effects and delay effects in the stability analysis of discrete neural networks. Impulsive differential equations, which are mathematical models for continuous-time dynamical systems with impulsive perturbations, have been successfully applied to many practical problems, see, e.g., Refs. [19]-[24]. Similarly, impulsive difference equations are suitable mathematical tools to model impulsive discretetime neural networks. Continuous-time neural networks with impulsive perturbations have been reported (see e.g. [25]-[28]). In [25], global stability properties have been analyzed for impulsive Hopfield-type neural networks whose impulses contain both the functional term and its integral. Sufficient conditions are derived in [26] based on vector Lyapunov functions and the M-matrix theory for ensuring global exponential stability of the neural networks with impulsive effects. In addition, the estimated exponential convergence rate is given as well. Exponential stability analysis of impulsive delay neural networks has been investigated in [27] and [28] based on the M-matrix theory and an impulsive delay differential inequality. However, it appears that little attention is devoted to the investigation of stability for discrete-time neural networks with time delays subject to impulsive perturbations, although such neural networks are important in the fields of natural sciences and applied technology. This motivates our study. 
In this paper, we shall deal with a class of discrete-time neural networks with time-varying delays subject to impulsive perturbations. Firstly, by utilizing the Lyapunov stability theory and discrete-time Halanay-type inequality, we shall establish some sufficient conditions for global exponential stability of discrete-time neural networks with time-varying delays. Secondly, we shall apply the results obtained to impulsive discrete-time neural networks and obtain new global exponential stability criteria and new estimated exponential convergence rate for impulsive discrete-time neural networks with time-varying delays.

The main contributions of the current paper include: (i) some new global exponential stability criteria are derived by means of the discrete-time Halanay-type inequality; (ii) the form of impulsive perturbations is more general than the existing ones in the literature which are described by either a linear matrix function or a simple nonlinear matrix function; and (iii) new global exponential stability criteria and convergence rate of impulsive neural networks with time-varying delays are obtained.

The rest of this paper is organized as follows. In Section II, impulsive discrete-time neural networks with time-varying delays are introduced and some preliminary lemmas are presented. In Section III, based on the Lyapunov stability theory and the discrete-time Halanay-type inequality, global exponential stability criteria are derived for discrete-time neural networks with timevarying delays for the case when the neural networks are free of impulsive perturbations as well as the case when they are subject to impulsive perturbations. Moreover, numerical examples are presented in Sections IV. Section V concludes the paper.

\section{Preliminaries And Problem Statement}

Consider the following impulsive neural network with time-varying delays:

$$
\left\{\begin{aligned}
u_{i}(m+1) & =a_{i} u_{i}(m)+\sum_{j=1}^{n} T_{i j} \hat{f}_{j}\left(u_{j}\left(m-\tau_{i j}(m)\right)\right)+I_{i}, & & m \neq n_{k}, \\
\Delta u_{i}(m) & =\hat{g}_{i}^{(k)}\left(m, u_{1}(m), \cdots, u_{n}(m)\right), & & m=n_{k}, \quad m \in N(1), \\
u_{i}(m) & =\phi_{i}(m), & & m \in N(-\tau, 0),
\end{aligned}\right.
$$

where $u_{i}(t)$ denotes the state of the $i$ th neuron at time $t ; a_{i} \in[0,1), i \in N(1, n)$, represents the passive decay rate, where $N(k)=\{k, k+1, k+2, \cdots\}, N(k, l)=\{k, k+1, k+2, \cdots, l\} ; \hat{f}_{j}$ is the neuron output signal function which is a continuous function; $T_{i_{j}}, \tau_{i j}(m) \geq 0$ denote, respectively, the connection weight and the transmission delay from the neuron $j$ to the neuron 
$i$ with $\tau=\max _{i, j \in N(1, n)}\left\{\tau_{i j}(m)\right\}$ and $m-\tau_{i j}(m) \rightarrow \infty$ as $m \rightarrow \infty ; f_{j}: R \rightarrow R$ is the neuron activation function with $f_{j}(0)=0$; and $I_{i}$ is the exogenous input, where $i \in N(1, n) . u_{i}(m)=$ $\phi_{i}(m), m \in N(-\tau, 0)$, is the initial condition for (1), where $\phi_{i}: N(-\tau, 0) \rightarrow R, i \in N(1, n)$, is a continuous function. A vector $u^{*}=\left[u_{1}^{*}, u_{2}^{*}, \cdots, u_{n}^{*}\right]^{T}$ is said to be an equilibrium point of the impulsive discrete-time neural network (1) if it satisfies

$$
u_{i}^{*}=a_{i} u_{i}^{*}+\sum_{j=1}^{n} T_{i j} \hat{f}_{j}\left(u_{j}^{*}\right)+I_{i} .
$$

Let $u_{i}^{*}$ be an equilibrium point of (1). For the purpose of brevity, we can shift the equilibrium $u_{i}^{*}$ to the origin by setting $x_{i}(m)=u_{i}(m)-u_{i}^{*}, i \in N(1, n)$. Then, the neural network (1) is transformed into

$$
\left\{\begin{aligned}
x_{i}(m+1) & =a_{i} x_{i}(m)+\sum_{j=1}^{n} T_{i j} f_{j}\left(x_{j}\left(m-\tau_{i j}(m)\right)\right), & & m \neq n_{k}, \\
\Delta x_{i}(m) & =g_{i}^{(k)}\left(m, x_{1}(m), \cdots, x_{n}(m)\right), & & m=n_{k}, \quad m \in N(1), \\
x_{i}(m) & =\phi_{i}(m), & & m \in N(-\tau, 0),
\end{aligned}\right.
$$

where

$$
f_{j}\left(x_{j}\left(m-\tau_{i j}(m)\right)\right)=\hat{f}_{j}\left(u_{j}\left(m-\tau_{i j}(m)\right)\right)-\hat{f}_{j}\left(u_{j}^{*}\left(m-\tau_{i j}(m)\right)\right)
$$

and

$$
g_{i}^{(k)}\left(m, x_{1}(m), \cdots, x_{n}(m)\right)=\hat{g}_{i}^{(k)}\left(m, u_{1}(m), \cdots, u_{n}(m)\right)-\hat{g}_{i}^{(k)}\left(m, u_{1}^{*}(m), \cdots, u_{n}^{*}(m)\right) .
$$

Without loss of generality, we may assume that the sequence $\left\{n_{k}, g_{k}^{(k)}\right\}$ of the impulsive effects satisfies the following assumptions.

Assumption 1: The sequence $\left\{n_{k}\right\}$ of the impulsive time points satisfies $n_{k} \in N(1), n_{k}+2 \leq$ $n_{k+1}$ and $\lim _{k \rightarrow \infty} n_{k}=\infty$ with $k \in N(0)$.

Assumption 2: For the impulsive increment function sequence $g_{k}^{(k)}$, there exists $\omega_{i j}^{(k)} \geq 0$ such that $\forall\left(x_{1}(t), x_{2}(t), \cdots, x_{n}(t)\right) \in R^{n}, t \in \in N(0)$, the following condition is satisfied,

$$
\left|x_{i}(t)+g_{i}^{(k)}\left(t, x_{1}(t), \cdots, x_{n}(t)\right)\right| \leq \sum_{j=1}^{n} \omega_{i j}^{(k)}\left|x_{j}(t)\right|, j \in N(1, n) .
$$

Clearly, the stability properties of the impulsive neural network (1) are equivalent to the stability properties of the impulsive neural network (2). Furthermore, we need the following definitions and lemmas. 
Definition 1: For the impulsive discrete-time neural network (2), the trivial equilibrium point is uniformly stable if there exists a positive constant $\varepsilon>0$, and $\max _{s \in N(-\tau, 0)}\{\|x(s)\|\} \leq \delta(\varepsilon)$, then, for any given $\delta(\varepsilon)>0,\|x(m)\|<\varepsilon, \forall m \in N(1)$.

Definition 2: For the impulsive discrete-time neural network (2), the trivial equilibrium point is asymptotically stable if the neural network (2) is uniformly stable and the following condition is satisfied,

$$
\lim _{m \rightarrow+\infty}\|x(m)\|=0 .
$$

Definition 3: For the impulsive discrete-time neural network (2), the trivial equilibrium point is exponentially stable if there exist positive constants $\kappa>0$ and $r \in(0,1)$ such that

$$
\|x(m)\| \leq \kappa r^{m}, \forall m \in N(1)
$$

where $r$ is called the exponential convergence rate. If (5) is satisfied for any initial condition $x(m) \in R^{n}, m \in N(-\tau, 0)$, the trivial equilibrium point is globally exponentially stable for the impulsive neural network (2).

Lemma 1: [29] (Discrete-time Halanay-type Inequality) Consider the following discrete-time system

$$
\Delta x(m)=f(m, x(m), x(m-1), \ldots, x(m-\tau))
$$

where $\Delta x(m)=x(m+1)-x(m)$ and $N \times R^{\tau+1} \rightarrow R$. The initial condition is given that $\phi_{i}: N(-\tau, 0) \rightarrow R, i \in N(1, n)$. Suppose that the real numbers sequence $\left\{b_{n}\right\}_{n \geq-\tau}$ is such that

$$
\Delta x_{n}=-a x_{n}+g\left(n, x_{n}, x_{n-1}, \cdots_{, n-\tau}\right), n \in N(0), a \in(0,1]
$$

and that there exists a $b \in(0, a)$ such that

$$
\Delta b_{n} \leq-a b_{n}+b \max _{i \in N(n-h, n)}\left\{b_{i}\right\}, \forall n \in N(0) .
$$

Then, there exists a $\lambda \in(0,1)$ such that

$$
b_{n} \leq \lambda^{n} \max _{i \in N(-h, 0)}\left\{b_{i}\right\}, \forall n \in N(0),
$$

where $\Delta b_{n}=b_{n+1}-b_{n}, h \in N(0)$, is a constant, $g: N(0) \times R^{h+1} \rightarrow R,\left(b_{-h}, b_{-h+1}, \cdots, b_{0}\right)$ is the initial condition and $\lambda$ is the smallest root in the interval $(0,1)$ of the following equation

$$
\lambda^{h+1}+(a-1) \lambda^{h}-b=0
$$




\section{MAIN RESULTS}

In this section, we shall firstly establish sufficient conditions for global exponential stability of discrete-time neural networks with time-varying delays. On the basis of the obtained results, we shall investigate the global exponential stability criteria and the estimated exponential convergence rate of impulsive discrete-time neural networks.

\section{A. Discrete-time Neural Networks}

Consider a discrete-time neural network described by

$$
\begin{aligned}
x_{i}(m+1) & =a_{i} x_{i}(m)+\sum_{j=1}^{n} T_{i j} f_{j}\left(x_{j}\left(m-\tau_{i j}(m)\right)\right), m \in N(1), \\
x_{i}(m) & =\phi_{i}(m), m \in N(-\tau, 0), i \in N(1, n) .
\end{aligned}
$$

In the following theorem, the results on the global exponential stability of the neural network (6) are presented.

Theorem 1: Suppose that Assumption 1, Assumption 2 and the following conditions are satisfied.

i) There exists a constant $\delta_{j}>0$ such that $\forall t_{1}, t_{2} \in N(1)$, the neuron activation function $f_{j}(m)$ in (1) is bounded and satisfies the following Lipschitz condition

$$
\left|f_{j}\left(t_{1}\right)-f_{j}\left(t_{2}\right)\right| \leq \delta_{j}\left|t_{1}-t_{2}\right|, \quad j \in N(1, n) .
$$

ii)

$$
a+\delta<1
$$

where $a=\max _{i \in N(1, n)}\left\{a_{i}\right\}$ and $\delta=\max _{i \in N(1, n)}\left\{\sum_{j=1}^{n}\left|T_{i j}\right| \delta_{j}\right\}$.

Then, the trival equilibrium point of (6) is globally exponentially stable with the convergence rate $\lambda$ which is the smallest root in the interval $(0,1)$ of the following equation

$$
\lambda^{\tau+1}-a \lambda^{\tau}-\delta=0 .
$$

Proof: From the trajectory $\{x(m)\}, m \in N(1)$, of system (6), we have

$$
x_{i}(m)=a_{i}^{m} x_{i}(0)+\sum_{s=0}^{m-1} a_{i}^{m-1-s} \sum_{j=1}^{n} T_{i j} f_{j}\left(x_{j}\left(s-\tau_{i j}(s)\right)\right), m \in N(1), i \in N(1, n) .
$$


Clearly,

$$
\left|x_{i}(m)\right| \leq a_{i}^{m}\left|x_{i}(0)\right|+\sum_{s=0}^{m-1} a_{i}^{m-1-s} \sum_{j=1}^{n}\left|T_{i j}\right|\left|f_{j}\left(x_{j}\left(s-\tau_{i j}(s)\right)\right)\right| .
$$

Then, by the Lipschitz condition (7), it follows from (10) that

$$
\begin{aligned}
\left|x_{i}(m)\right| \leq & a_{i}^{m} \max _{i \in N(1, n)}\left\{\left|x_{i}(0)\right|\right\}+\sum_{s=0}^{m-1} a_{i}^{m-1-s} \sum_{j=1}^{n}\left|T_{i j}\right| \delta_{j}\left|x_{j}\left(s-\tau_{i j}(s)\right)\right| \\
\leq & a_{i}^{m} \max _{i \in N(1, n)}\left\{\left|x_{i}(0)\right|\right\}+\sum_{s=0}^{m-1} a_{i}^{m-1-s} \sum_{j=1}^{n}\left|T_{i j}\right| \delta_{j} \max _{j \in N(1, n)}\left\{\max _{t \in N(s-\tau, s)}\left\{\left|x_{j}(t)\right|\right\}\right\} \\
\leq & a_{i}^{m} \max _{i \in N(1, n)}\left\{\left|x_{i}(0)\right|\right\} \\
& +\sum_{s=0}^{m-1} a_{i}^{m-1-s} \max _{i \in N(1, n)}\left\{\sum_{j=1}^{n}\left|T_{i j}\right| \delta_{j}\right\} \max _{t \in N(s-\tau, s)}\left\{\max _{j \in N(1, n)}\left\{\left|x_{j}(t)\right|\right\}\right\} .
\end{aligned}
$$

For any $m \in N(-\tau)$, let $\delta=\max _{i \in N(1, n)}\left\{\sum_{j=1}^{n}\left|T_{i j}\right| \delta_{j}\right\}$ and

$$
\xi_{m}=\left\{\begin{array}{l}
\max _{i \in N(1, n)}\left\{\left|x_{i}(m)\right|\right\}, m \in N(-\tau, 0), \\
a_{i}^{m} \max _{i \in N(1, n)}\left\{\left|x_{i}(0)\right|\right\}+\delta \sum_{s=0}^{m-1} a_{i}^{m-1-s} \max _{t \in N(s-\tau, s)}\left\{\max _{j \in N(1, n)}\left\{\left|x_{j}(t)\right|\right\}\right\}, m \in N(1) .
\end{array}\right.
$$

Then, (11) is reduced to

$$
\left|x_{i}(m)\right| \leq \xi_{m}, i \in N(1, n) .
$$

Since

$$
\begin{aligned}
\Delta \xi_{m}=\xi_{m+1}-\xi_{m} & =-(1-a) \xi_{m}+\delta \max _{t \in N(m-\tau, m)}\left\{\max _{j \in N(1, n)}\left\{\left|x_{j}(t)\right|\right\}\right\} \\
\leq & -(1-a) \xi_{m}+\delta \max _{t \in N(m-\tau, m)}\left\{\xi_{t}\right\}, \forall m \in N(1),
\end{aligned}
$$

it follows from Lemma 1 that there exists a $\lambda \in(0,1)$ such as

$$
\xi_{m} \leq \lambda^{m} \max _{t \in N(-\tau, 0)}\left\{\xi_{t}\right\}, \forall m \in N(0)
$$

Furthermore, by (12), we have

$$
\|x(m)\|_{\infty}=\max _{i \in N(1, n)}\left\{\left|x_{i}(m)\right|\right\} \leq \xi_{m}, \forall m \in N(-\tau) .
$$

Thus,

$$
\|x(m)\|_{\infty} \leq \max _{t \in N(-\tau, 0)}\left\{\xi_{t}\right\} \lambda^{m}, \forall m \in N(0)
$$


Therefore, by virtue of Definition 3, the trivial equilibrium point of (6) is globally exponentially stable with the convergence rate $\lambda$ which is the smallest root in the interval $(0,1)$ of the following equation

$$
\lambda^{\tau+1}-a \lambda^{\tau}-\delta=0
$$

This completes the proof.

\section{B. Impulsive Discrete-time Neural Networks}

We now give sufficient conditions for global exponential stability of impulsive discrete-time neural network (2). In addition, its convergence rate will also be presented.

Theorem 2: Suppose that Assumption 1, Assumption 2 and the following conditions are satisfied.

i) There exists a constant $\delta_{j}>0$ such that $\forall t_{1}, t_{2} \in N(1)$, the neuron activation function $f_{j}(m)$ in (1) is bounded and satisfies the following Lipschitz condition

$$
\left|f_{j}\left(t_{1}\right)-f_{j}\left(t_{2}\right)\right| \leq \delta_{j}\left|t_{1}-t_{2}\right|, \quad j \in N(1, n)
$$

ii)

$$
\sum_{j=0}^{k} \ln l_{j}-(k+1) \ln a \leq 0, \quad k \in N(0)
$$

iii)

$$
a+\delta<1
$$

where $a=\max _{i \in N(1, n)}\left\{a_{i}\right\}, \delta=\max _{i \in N(1, n)}\left\{\sum_{j=1}^{n}\left|T_{i j}\right| \delta_{j}\right\}$ and $l_{k}=\max _{i \in N(1, n)}\left\{\sum_{j=1}^{n} \omega_{i j}^{(k)}\right\}$.

Then, the equilibrium point $u_{i}^{*}$ of (1) is globally exponentially stable with the convergence rate $\lambda$, which is the smallest root in the interval $(0,1)$ of the following equation

$$
\lambda^{\tau+1}-a \lambda^{\tau}-\delta=0 .
$$

Proof: For any $m \in\left(N_{k}, N_{k+1}\right]$, we have

$$
\left|x_{i}(m)\right| \leq a_{i}^{m-N_{k}-1}\left|x_{i}\left(N_{k}+1\right)\right|+\sum_{s=N_{k}+1}^{m-1} a_{i}^{m-1-s} \sum_{j=1}^{n}\left|T_{i j}\right|\left|f_{j}\left(x_{j}\left(s-\tau_{i j}(s)\right)\right)\right| .
$$


Applying (3) and (15) to (19) yields

$$
\begin{aligned}
\left|x_{i}(m)\right| \leq & a_{i}^{m-N_{k}-1} \sum_{j=1}^{n} \omega_{i j}^{(k)}\left|x_{j}\left(N_{k}\right)\right|+\sum_{s=N_{k}+1}^{m-1} a_{i}^{m-1-s} \sum_{j=1}^{n}\left|T_{i j}\right| \delta_{j}\left|x_{j}\left(s-\tau_{i j}(s)\right)\right| \\
\leq & a_{i}^{m-N_{k}-1} \sum_{j=1}^{n} \omega_{i j}^{(k)} \max _{j \in N(1, n)}\left\{\left|x_{j}\left(N_{k}\right)\right|\right\} \\
& +\sum_{s=N_{k}+1}^{m-1} a_{i}^{m-1-s} \sum_{j=1}^{n}\left|T_{i j}\right| \delta_{j} \max _{j \in N(1, n)}\left\{\left|x_{j}\left(s-\tau_{i j}(s)\right)\right|\right\}
\end{aligned}
$$

Let $\delta=\max _{i \in N(1, n)}\left\{\sum_{j=1}^{n}\left|T_{i j}\right| \delta_{j}\right\}$ and $l_{k}=\max _{i \in N(1, n)}\left\{\sum_{j=1}^{n} \omega_{i j}^{(k)}\right\}$. Then, it follows from (20) that

$$
\left|x_{i}(m)\right| \leq a^{m-N_{k}-1} l_{k} \max _{j \in N(1, n)}\left\{\left|x_{j}\left(N_{k}\right)\right|\right\}+\delta \sum_{s=N_{k}+1}^{m-1} a^{m-1-s} \max _{t \in N(s-\tau, s)}\left\{\max _{j \in N(1, n)}\left\{\left|x_{j}(t)\right|\right\}\right.
$$

Thus,

$\left|x_{i}\left(N_{k+1}\right)\right| \leq a^{N_{k+1}-N_{k}-1} l_{k} \max _{j \in N(1, n)}\left\{\left|x_{j}\left(N_{k}\right)\right|\right\}+\delta \sum_{s=N_{k}+1}^{N_{k+1}-1} a^{N_{k+1}-1-s} \max _{t \in N(s-\tau, s)}\left\{\max _{j \in N(1, n)}\left\{\left|x_{j}(t)\right|\right\}\right\}$.

By induction, we obtain that

$$
\begin{aligned}
\left|x_{i}\left(N_{k}\right)\right| \leq & a^{N_{k}-N_{0}-k} \prod_{j=0}^{k-1} l_{j} \max _{j \in N(1, n)}\left\{\left|x_{j}\left(N_{0}\right)\right|\right\} \\
& +\delta \prod_{j=1}^{k-1} l_{j} \sum_{s=N_{0}+1}^{N_{1}-1} a^{N_{1}-k-s} \max _{t \in N(s-\tau, s)}\left\{\max _{j \in N(1, n)}\left\{\left|x_{j}(t)\right|\right\}\right\} \\
& +\delta \prod_{j=2}^{k-1} l_{j} \sum_{s=N_{1}+1}^{N_{2}-1} a^{N_{k}-k-s+1} \max _{t \in N(s-\tau, s)}\left\{\max _{j \in N(1, n)}\left\{\left|x_{j}(t)\right|\right\}\right\}+\cdots \\
& +\delta l_{k-1} \sum_{s=N_{k-2}+1}^{N_{k-1}-1} a^{N_{k}-s-2} \max _{t \in N(s-\tau, s)}\left\{\max _{j \in N(1, n)}\left\{\left|x_{j}(t)\right|\right\}\right\} \\
& +\delta \sum_{s=N_{k-1}+1}^{N_{k}-1} a^{N_{k}-s-1} \max _{t \in N(s-\tau, s)}\left\{\max _{j \in N(1, n)}\left\{\left|x_{j}(t)\right|\right\}\right\} .
\end{aligned}
$$

Since

$$
\begin{aligned}
\left|x_{i}\left(N_{k}\right)\right| \leq & a^{N_{k}-N_{0}-k} \prod_{j=0}^{k-1} l_{j} \max _{j \in N(1, n)}\left\{\left|x_{j}\left(N_{0}\right)\right|\right\} \\
& +\delta \prod_{j=1}^{k-1} l_{j} \sum_{s=N_{0}+1}^{N_{1}-1} a^{N_{1}-k-s} \max _{t \in N(s-\tau, s)}\left\{\max _{j \in N(1, n)}\left\{\left|x_{j}(t)\right|\right\}\right\} \\
& +\delta \prod_{j=2}^{k-1} l_{j} \sum_{s=N_{1}+1}^{N_{2}-1} a^{N_{k}-k-s+1} \max _{t \in N(s-\tau, s)}\left\{\max _{j \in N(1, n)}\left\{\left|x_{j}(t)\right|\right\}\right\}+\cdots
\end{aligned}
$$




$$
\begin{aligned}
& +\delta l_{k-1} \sum_{s=N_{k-2}+1}^{N_{k-1}-1} a^{N_{k}-s-2} \max _{t \in N(s-\tau, s)}\left\{\max _{j \in N(1, n)}\left\{\left|x_{j}(t)\right|\right\}\right\} \\
& +\delta \sum_{s=N_{k-1}+1}^{N_{k}-1} a^{N_{k}-s-1} \max _{t \in N(s-\tau, s)}\left\{\max _{j \in N(1, n)}\left\{\left|x_{j}(t)\right|\right\}\right\},
\end{aligned}
$$

we have

$$
\begin{aligned}
\left|x_{i}\left(N_{k}\right)\right| \leq & a^{N_{k}-k} \prod_{j=0}^{k-1} l_{j} \max _{j \in N(1, n)}\left\{\left|x_{j}(0)\right|\right\} \\
& +\delta \prod_{j=0}^{k-1} l_{j} \sum_{s=0}^{N_{0}-1} a^{N_{k}-k-s-1} \max _{t \in N(s-\tau, s)}\left\{\max _{j \in N(1, n)}\left\{\left|x_{j}(t)\right|\right\}\right\} \\
& +\delta \prod_{j=1}^{k-1} l_{j} \sum_{s=N_{0}+1}^{N_{1}-1} a^{N_{1}-k-s} \max _{t \in N(s-\tau, s)}\left\{\max _{j \in N(1, n)}\left\{\left|x_{j}(t)\right|\right\}\right\} \\
& +\delta \prod_{j=2}^{k-1} l_{j} \sum_{s=N_{1}+1}^{N_{2}-1} a^{N_{k}-k-s+1} \max _{t \in N(s-\tau, s)}\left\{\max _{j \in N(1, n)}\left\{\left|x_{j}(t)\right|\right\}\right\}+\cdots \\
& +\delta l_{k-1} \sum_{s=N_{k-2}+1}^{N_{k-1}-1} a^{N_{k}-s-2} \max _{t \in N(s-\tau, s)}\left\{\max _{j \in N(1, n)}\left\{\left|x_{j}(t)\right|\right\}\right\} \\
& +\delta \sum_{s=N_{k-1}+1}^{N_{k}-1} a^{N_{k}-s-1} \max _{t \in N(s-\tau, s)}\left\{\max _{j \in N(1, n)}\left\{\left|x_{j}(t)\right|\right\}\right\} .
\end{aligned}
$$

Thus, it follows from (21) and (22) that

$$
\begin{aligned}
\left|x_{i}(m)\right| \leq & a^{m-k-1} \prod_{j=0}^{k} l_{j} \max _{j \in N(1, n)}\left\{\left|x_{j}(0)\right|\right\} \\
& +\delta \prod_{j=0}^{k} l_{j} \sum_{s=0}^{N_{0}-1} a^{m-k-s-2} \max _{t \in N(s-\tau, s)}\left\{\max _{j \in N(1, n)}\left\{\left|x_{j}(t)\right|\right\}\right\} \\
& +\delta \prod_{j=1}^{k} l_{j} \sum_{s=N_{0}+1}^{N_{1}-1} a^{m-k-s-1} \max _{t \in N(s-\tau, s)}\left\{\max _{j \in N(1, n)}\left\{\left|x_{j}(t)\right|\right\}\right\}+\cdots \\
& +\delta l_{k} \sum_{s=N_{k-1}+1}^{N_{k}-1} a^{m-s-2} \max _{t \in N(s-\tau, s)}\left\{\max _{j \in N(1, n)}\left\{\left|x_{j}(t)\right|\right\}\right\} \\
& +\delta \sum_{s=N_{k}+1}^{m-1} a^{m-1-s} \max _{t \in N(s-\tau, s)}\left\{\max _{j \in N(1, n)}\left\{\left|x_{j}(t)\right|\right\}\right\} .
\end{aligned}
$$

By (21) and (23), we obtain

$$
\left|x_{i}(m)\right| \leq a^{m} \max _{j \in N(1, n)}\left\{\left|x_{j}(0)\right|\right\}+\delta \sum_{s=0}^{N_{0}-1} a^{m-s-1} \max _{t \in N(s-\tau, s)}\left\{\max _{j \in N(1, n)}\left\{\left|x_{j}(t)\right|\right\}\right\}
$$




$$
\begin{aligned}
& +\delta \sum_{s=N_{0}+1}^{N_{1}-1} a^{m-s-1} \max _{t \in N(s-\tau, s)}\left\{\max _{j \in N(1, n)}\left\{\left|x_{j}(t)\right|\right\}\right\}+\cdots \\
& +\delta \sum_{s=N_{k-1}+1}^{N_{k}-1} a^{m-s-1} \max _{t \in N(s-\tau, s)}\left\{\max _{j \in N(1, n)}\left\{\left|x_{j}(t)\right|\right\}\right\} \\
& +\delta \sum_{s=N_{k}+1}^{m-1} a^{m-1-s} \max _{t \in N(s-\tau, s)}\left\{\max _{j \in N(1, n)}\left\{\left|x_{j}(t)\right|\right\}\right\} \\
& =\eta_{m}, \forall i \in N(1, n), m \in N(1) .
\end{aligned}
$$

Let

$$
\xi_{m}=\left\{\begin{array}{l}
\max _{i \in N(1, n)}\left\{\left|x_{i}(m)\right|\right\}, m \in N(-\tau, 0), \\
c_{m}, m \in N(1) .
\end{array}\right.
$$

Then, the rest of the proof follows readily from similar arguments as those given for the proof of Theorem 1. This completes the proof.

Corollary 1: Suppose that Assumption 1, Assumption 2 and the following conditions are satisfied.

i) There exists a constant $\delta_{j}>0$ such that $\forall t_{1}, t_{2} \in R$, the neuron activation function $f_{j}(m)$ in (1) is bounded and satisfies the following Lipschitz condition

$$
\left|f_{j}\left(t_{1}\right)-f_{j}\left(t_{2}\right)\right| \leq \delta_{j}\left|t_{1}-t_{2}\right|, \quad j \in N(1, n)
$$

ii)

$$
\eta+\delta<1
$$

where $\eta=\sup _{k \in N(0)}\left\{a, l_{k}\right\}, a=\max _{i \in N(1, n)}\left\{a_{i}\right\}, \delta=\max _{i \in N(1, n)}\left\{\sum_{j=1}^{n}\left|T_{i j}\right| \delta_{j}\right\}$ and $l_{k}=\max _{i \in N(1, n)}\left\{\sum_{j=1}^{n} \omega_{i j}^{(k)}\right\}$. Then, the equilibrium point $u_{i}^{*}$ of (1) is globally exponentially stable with the convergence rate $\lambda$, which is the smallest root in the interval $(0,1)$ of the following equation

$$
\lambda^{\tau+1}-\eta \lambda^{\tau}-\delta=0 .
$$

Corollary 2: Suppose that Assumption 1, Assumption 2 and the following conditions are satisfied.

i) There exists a constant $\delta_{j}>0$ such that $\forall t_{1}, t_{2} \in N(1)$, the neuron activation function $f_{j}(m)$ in (1) is bounded and satisfies the following Lipschitz condition

$$
\left|f_{j}\left(t_{1}\right)-f_{j}\left(t_{2}\right)\right| \leq \delta_{j}\left|t_{1}-t_{2}\right|, \quad j \in N(1, n)
$$


ii)

$$
\beta<\frac{1}{2}
$$

where $\beta=\sup _{k \in N(0)}\left\{a, \delta, l_{k}\right\}, a=\max _{i \in N(1, n)}\left\{a_{i}\right\}, \delta=\max _{i \in N(1, n)}\left\{\sum_{j=1}^{n}\left|T_{i j}\right| \delta_{j}\right\}$ and $l_{k}=\max _{i \in N(1, n)}\left\{\sum_{j=1}^{n} \omega_{i j}^{(k)}\right\}$. Then, the equilibrium point $u_{i}^{*}$ of (1) is globally exponentially stable with the convergence rate $\lambda$, which is the smallest root in the interval $(0,1)$ of the following equation

$$
\lambda^{\tau+1}-\eta \lambda^{\tau}-\delta=0 .
$$

\section{NUMERICAL EXAMPLES}

In this section, two numerical examples are presented to verify and illustrate the usefulness of our main results.

Example 1 In this example, we consider a two-neuron discrete-time neural network with time delays

$$
\begin{gathered}
\left\{\begin{array}{l}
x_{1}(m+1)=\frac{1}{2} x_{1}(m)+\frac{1}{8} f_{1}\left(x_{1}(m-1)\right)-\frac{1}{4} f_{2}\left(x_{2}(m-1)\right), \\
x_{2}(m+1)=\frac{1}{3} x_{2}(m)+\frac{1}{8} f_{1}\left(x_{1}(m-1)\right)+\frac{1}{3} f_{2}\left(x_{2}(m-1)\right),
\end{array} \quad(m \in N(1))\right. \\
\left\{\begin{array}{l}
x_{1}(m)=\phi_{1}(m), \\
x_{2}(m)=\phi_{2}(m),
\end{array}(m \in N(-1,0))\right.
\end{gathered}
$$

where $f_{1}(t)=\sin t, f_{2}(t)=t, \phi_{1}(t)=t^{2}, \phi_{2}(t)=-t^{3}$. It is easy to verify that

$$
\begin{gathered}
\left|f_{1}(s)-f_{1}(t)\right| \leq|s-t|, \forall s, t \in R, \\
\left|f_{2}(s)-f_{2}(t)\right| \leq|s-t|, \forall s, t \in R, \\
\max _{i \in N(1,2)}\left\{a_{i}\right\}+\max _{i \in N(1,2)}\left\{\sum_{j=1}^{2}\left|T_{i j}\right| \delta_{j}\right\}=0.9583<1 .
\end{gathered}
$$

Thus, all the conditions of Theorem 1 are satisfied. Therefore, the trivial equilibrium point of (2) is globally exponentially stable with the convergence rate $\lambda=0.9717$.

Example 2 In this example, we consider a three-neuron impulsive discrete-time neural network with time delays

$$
\left\{\begin{array}{l}
x_{1}(m+1)=\frac{1}{2} x_{1}(m)+\frac{1}{8} f_{1}\left(x_{1}(m-2)\right)-\frac{1}{4} f_{2}\left(x_{2}(m-1)\right)+\frac{1}{16} f_{3}\left(x_{3}(m-1)\right)+1, \\
x_{2}(m+1)=\frac{1}{3} x_{2}(m)+\frac{1}{4} f_{1}\left(x_{1}(m-2)\right)+\frac{1}{8} f_{2}\left(x_{2}(m-1)\right)+2, \\
x_{3}(m+1)=\frac{1}{4} x_{3}(m)+\frac{1}{16} f_{1}\left(x_{1}(m-2)\right)-\frac{1}{8} f_{2}\left(x_{2}(m-1)\right)+\frac{1}{16} f_{3}\left(x_{3}(m-1)\right)+1,
\end{array}\right.
$$




$$
\begin{aligned}
& \left\{\begin{aligned}
& \Delta x_{1}(m)=-\frac{2}{3} x_{1}(m), \\
& \Delta x_{2}(m)=-\frac{2}{3} x_{2}(m), \quad(m=4 k, m \in N(1), k \in N(1)) \\
& \Delta x_{3}(m)=-\frac{2}{3} x_{3}(m), \\
&\left\{\begin{array}{l}
x_{1}(m)=\phi_{1}(m), \\
x_{2}(m)=\phi_{2}(m), \\
x_{3}(m)=\phi_{3}(m),
\end{array}\right.
\end{aligned}\right.
\end{aligned}
$$

where $f_{1}(t)=f_{3}(t)=\sin t, f_{2}(t)=t, \phi_{1}(t)=\phi_{2}(t)=\phi_{3}(t)=-t^{3}+2$. Here, for computational convenience, we assume that the neural network is only subject to linear impulsive perturbations. It can be shown that the equilibrium point of the impulsive discrete-time neural network (1) is

$$
x^{*}=\left(x_{1}^{*}, x_{2}^{2}, x_{3}^{*}\right)^{T}=(1.5786,1.8355,1.2322)^{T} .
$$

In addition, one can easily check that

$$
\begin{gathered}
\left|f_{1}(s)-f_{1}(t)\right| \leq|s-t|, \forall s, t \in R, \\
\left|f_{2}(s)-f_{2}(t)\right| \leq|s-t|, \forall s, t \in R, \\
\left|f_{3}(s)-f_{3}(t)\right| \leq|s-t|, \forall s, t \in R, \\
\sum_{j=0}^{k} \ln l_{j}-(k+1) \ln a=-0.4055 \leq 0, \quad k \in N(0), \\
\max _{i \in N(1,2)}\left\{a_{i}\right\}+\max _{i \in N(1,2)}\left\{\sum_{j=1}^{2}\left|T_{i j}\right| \delta_{j}\right\}=0.8750<1 .
\end{gathered}
$$

Thus, all the conditions of Theorem 2 are satisfied. Therefore, the equilibrium point of (1) is globally exponentially stable with the convergence rate $\lambda=0.9319$.

In conclusion, it is clear that all the state variables in both examples globally exponentially converge to their equilibrium points. 


\section{CONCLUSION}

We have developed a set of computable sufficient conditions for global exponential stability of discrete-time neural networks with time delays based on the Lyapunov stability theory and a discrete-time Halanay-type inequality technique. Moreover, the obtained results were then applied to derive the global exponential stability criteria and its convergence rate for impulsive discrete-time neural networks with time delays. Finally, two numerical examples were given to show the effectiveness of our results.

\section{REFERENCES}

[1] S. Guo, L. Huang and L. Wang, "Exponential stability of discrete-time Hopfield neural networks," Comp. and Math. with Appl., vol. 47, pp. 1249-1256, 2004.

[2] J. Cao, P. Li, and W. Wang, "Global synchronization in arrays of delayed neural networks with constant and delayed coupling, Phys. Lett. A, vol. 353, pp. 318-325, 2006.

[3] H. Qiao, J. Peng, and Z. B. Xu, ”A reference model approach to stability analysis of neural networks," IEEE Trans. Syst. Man Cybern. B, Cybern., vol. 33, no. 6, pp. 925-936, Dec. 2003.

[4] H. Qiao, J. Peng, and Z. B. Xu, ”Nonlinear measures: A new approach to exponential stability analysis for Hopfield-type neural networks," IEEE Trans. Neural Netw., vol. 12, no. 2, pp. 360-370, Mar. 2001.

[5] J. Cao and Q. Song, ”Stability in Cohen-Grossberg-type bidirectional associative memory neural networks with time-varying delays," Nonlinearity, vol. 19, no. 7, pp. 1601-1617, 2006.

[6] J. Liang, J. Cao, and J. Lam, "Convergence of discrete-time recurrent neural networks with variable delay," Int. J. Bifurc. Chaos, vol. 15, no. 2, pp. 581-595, 2005.

[7] Y. Liu, Z.Wang, and X. Liu, "Synchronization and state estimation for discrete-time complex networks with distributed delays," IEEE Trans. Syst. Man Cybern. B, Cybern., vol. 38, no. 5, pp. 1314-1325, Oct. 2008.

[8] Y. Liu, Z. Wang, and X. Liu, "Global exponential stability of generalized recurrent neural networks with discrete and distributed delays," Neural Netw., vol. 19, pp. 667-675, 2006.

[9] Y. Shen and J. Wang, ”Noise-induced stabilization of the recurrent neural networks with mixed time-varying delays and Markovian- switching parameters," IEEE Trans. Neural Netw., vol. 18, no. 6, pp. 1857-1862, Nov. 2007.

[10] K. Gopalsamy and X. Z. He, "Delay-independent stability in bi-directional associative memory networks," IEEE Trans. Neural Netw., vol. 5, no. 6, pp. 998-1002, Nov. 1994.

[11] G. Chen, J. Zhou, and Z. Liu, "Global synchronization of coupled delayed neural networks and applications to chaotic CNN models," Int. J. Bifurc. Chaos, vol. 14, no. 7, pp. 2229-2240, 2004.

[12] W. Lu and T. Chen, "Synchronization of coupled connected neural networks with delays," IEEE Trans. Circuits Syst. I, Reg. Papers, vol. 51, no. 12, pp. 2491-2503, Dec. 2004.

[13] Q. Song and J. Cao, "Periodic solutions and its exponential stability of reaction-diffusion recurrent neural networks with continuously distributed delays," Nonlinear Anal.: Real World Appl., vol. 7, no. 1, pp. 65-80, 2006.

[14] J. Liang, J. Cao, and D. W. C. Ho, ’Discrete-time bidirectional associative memory neural networks with variable delays," Phys. Lett. A, vol. 335, no. 2-3, pp. 226-234, 2005. 
[15] S. Mohamad and K. Gopalsamy, "Exponential stability of continuous-time and discrete-time cellular neural networks with delays," Appl. Math. Comput., vol. 135, no. 1, pp. 17-38, 2003.

[16] W. Lu and T. Chen, "Synchronization analysis of linearly coupled networks of discrete time systems," Physica D, vol. 198, pp. 148-168, 2004.

[17] M. P. Casey, "The dynamics of discrete-time computation with application to recurrent neural networks and finite state machine extraction," Neural Comput., vol. 8, no. 6, pp. 1135-1178, 1996.

[18] W. Lu and T. Chen, "Global synchronization of discrete-time dynamical network with a directed graph," IEEE Trans. Circuits Syst. II, Exp. Briefs, vol. 54, no. 2, pp. 136-140, Feb. 2007.

[19] A. Anokhin, L. Berezansky, and E. Braverman, "Exponential stability of linear delay impulsive differential equations," $J$. Math. Anal. Appl., vol. 193, pp. 923-941, 1995.

[20] X. Z. Liu, "Stability results for impulsive differential systems with applications to population growth models," Dyn. Stability Syst., vol. 9, no. 2, pp. 163-174, 1994.

[21] J. Shen, Z. Luo, and X. Z. Liu, "Impulsive stabilization of functional differential equations via Liapunov functionals," $J$. Math. Anal. Appl., vol. 240, pp. 1-5, 1999.

[22] V. Lakshmikantham and X. Z. Liu, Stability Analysis in Terms of Two Measures. Singapore: World Scientific, 1993.

[23] V. Lakshmikantham, D. D. Bainov and P. S. Sineonov, Theory of Impulsive Differential Equations. Singapore: World Scientific, 1989.

[24] X. Z. Liu, "Stability results for impulsive differential systems with applications to population growth models," Dyn. Stability Syst., vol. 9, no. 2, pp. 163-174, 1994.

[25] H. Akca, R. Alassar, V. Covachev, Z. Covacheva, and E. Al-Zahrani, ”Continuous time additive Hopfield-type neural networks with impulses," J. Math. Anal. Appl., vol. 290, pp. 436-451, 2004.

[26] Q. Song and J. Cao, "Impulsive effects on stability of fuzzy Cohen-Grossberg neural networks with time-varying delays," IEEE Trans. Syst. Man Cybern. B, Cybern., vol. 37, no. 3, pp. 733-741, Jun. 2007.

[27] X. Z. Liu, K. L. Teo, and B. J. Xu, "Exponential stability of impulsive high-order Hopfield-type neural networks with time-varying delays," IEEE Trans. Neural Netw., vol. 16, no. 6, pp. 1329-1339, Nov. 2005.

[28] D. Y. Xu and Z. C. Yang, ”Stability analysis of delay neural networks with impulsive effects," IEEE Trans. Circuits Syst. I, Exp. Briefs, vol. 52, pp. 517-521, 2005.

[29] E. Liz and J. B. Ferreiro, ”A note on the global stability of generalized difference equations,” Appl. Math. Lett., vol. 15, pp. 655-659, 2002. 